University of Nebraska - Lincoln

DigitalCommons@University of Nebraska - Lincoln

CSE Conference and Workshop Papers

Computer Science and Engineering, Department

2000

\title{
Virtual Topology Reconfiguration of Wavelength-routed Optical WDM Networks
}

Byrav Ramamurthy

University of Nebraska-Lincoln, bramamurthy2@unl.edu

Ashok Ramakrishnan

University of Nebraska-Lincoln

Follow this and additional works at: https://digitalcommons.unl.edu/cseconfwork

Part of the Computer Sciences Commons

Ramamurthy, Byrav and Ramakrishnan, Ashok, "Virtual Topology Reconfiguration of Wavelength-routed Optical WDM Networks" (2000). CSE Conference and Workshop Papers. 84.

https://digitalcommons.unl.edu/cseconfwork/84

This Article is brought to you for free and open access by the Computer Science and Engineering, Department of at DigitalCommons@University of Nebraska - Lincoln. It has been accepted for inclusion in CSE Conference and Workshop Papers by an authorized administrator of DigitalCommons@University of Nebraska - Lincoln. 


\title{
Virtual Topology Reconfiguration of Wavelength-routed Optical WDM Networks
}

\author{
Byrav Ramamurthy and Ashok Ramakrishnan \\ Department of Computer Science and Engineering \\ University of Nebraska - Lincoln \\ Lincoln, NE 68588-0115, U.S.A. \\ E-mail: \{byrav, aramakri\}@cse.unl.edu
}

\begin{abstract}
The bandwidth requirements of the Internet are increasing every day and there are newer and more bandwidththirsty applications emerging on the horizon. Wavelength division multiplexing (WDM) is the next step towards leveraging the capabilities of the optical fiber, especially for wide-area backbone networks. The ability to switch a signal at intermediate nodes in a WDM network based on their wavelengths is known as wavelength-routing. One of the greatest advantages of using wavelength-routing WDM is the ability to create a virtual topology different from the physical topology of the underlying network. This virtual topology can be reconfigured when necessary, to improve performance. We discuss the previous work done on virtual topology design and also discuss and propose different reconfiguration algorithms applicable under different scenarios.
\end{abstract}

Keywords: Optical networks, wavelength division multiplexing (WDM), lightpath, reconfiguration, network control and management, and linear programming.

\section{Introduction}

Wavelength division multiplexing (WDM) is a promising approach for using the extensive bandwidth available in an optical communication medium [1], [4], [5]. The concept of multiple wavelengths carrying traffic belonging to different sessions along the same physical link is called wavelength division multiplexing. There has been significant research on realizing WDM over wide-area optical networks [2], [3].

In a WDM network, it is possible to route data to their respective destinations based on their wavelengths. The use of wavelength to route data is referred to as wavelength routing, and a network which employs this technique is known as a wavelength-routed network [4]. Such a network consists of wavelength-routing switches (or routing nodes) which are interconnected by optical fibers. Some routing nodes are attached to access stations where data from several end-users could be multiplexed on to a single WDM channel. An access station also provides optical-toelectronic $(\mathrm{O} / \mathrm{E})$ conversion and vice versa to interface the optical network with conventional electronic equipment. A wavelength-routed network which carries data from one access station to another without any intermediate $\mathrm{O} / \mathrm{E}$ conversion is referred to as an all-optical wavelength-routed network. Such all-optical wavelength-routed networks have been proposed for building large wide area networks. A sample optical wide-area network (WAN) is shown in Figure 1.

An optical network consists of nodes/switching elements connected by physical links i.e., optical fibers. This constitutes the physical topology of the optical network. A topology based on lightpaths ${ }^{1}$ established between pairs of nodes constitutes the virtual topology [3], [8]. A virtual path consists of a series of virtual links (lightpaths). Data is carried on such virtual paths between the source and the destination. The difference between a physical link, a lightpath and a virtual path is shown in Figure 2.

Design of efficient virtual topologies for wavelength-routed WDM networks is an important problem which has been addressed by several researchers [2], [3], [6]. However, when the traffic on the network changes, it is vital to transition from one virtual topology to another (using a reconfiguration procedure) in an efficient manner to minimize the disruption in the network (to be explained later). We address this important problem in this paper by proposing a new reconfiguration algorithm and compare it to previous approaches [6]. The rest of the paper is organized as follows. In Section 2, we survey the previous work on virtual topology design and reconfiguration of wavelength-routed WDM optical networks. In Sections 3 and 4 we describe the virtual topology design problem as a linear formulation. In Section 5, we present our proposed reconfiguration algorithm. In Section 6, we discuss the results obtained for sample network topologies. In Section 7, we present the conclusion.

\section{Previous Work}

Virtual (or logical) topology design for a wavelength division multiplexed (WDM) optical network has been studied by several researchers. Mainly, the design problem was seen from a LAN (broadcast) [7], [10], [17] or a WAN

\footnotetext{
${ }^{1}$ A lightpath is a virtual link, i.e., a sequence of physical links carrying a signal on the same wavelength from source to destination.
} 
(wavelength-routed) [2], [3], [6] point of view. In almost all of these studies the objective was to find the best virtual topology, for a given physical topology and a set of wavelength and transceiver constraints, that can accommodate the expected traffic in the network. There was also an early study [13] on embedding a regular topology over a given physical topology. The choice of the regular topology was made without considering the traffic demand. Broadcast networks can be further classified as single-hop or multihop depending on the logical connections. In a single-hop network, traffic from a source to destination is carried along a single lightpath all the way through. In a multi-hop network, traffic can go through one or more sequence of lightpaths before it reaches the destination.

\subsection{Wavelength-routed networks: Virtual topology design}

The problem of designing a virtual topology for wavelength-routed optical network has been studied by several researchers. As in the case with broadcast networks the virtual topology design problem can be approached in two ways. One is embedding of a regular graph (virtual topology) on to the physical topology based on the traffic. The other is determining the best virtual topology for the given traffic pattern.

The authors in [13] propose the Lightnet architecture, the principle of which is embedding a virtual topology in the wavelength domain over the physical topology taking into consideration the wavelength constraint, switch sizes and the average hop distance. Embedding a virtual topology over a physical topology consists of associating the nodes of the virtual topology with the nodes in the physical topology and realizing the edges of the virtual topology by creating lightpaths. Since the problem of embedding a virtual topology on a physical topology, while minimizing the number of wavelengths used, is an NP-complete problem, the authors have proposed a two-phase heuristic solution involving finding an equivalent representation for the physical topology as a string, and establishing a sequential mapping of the regular topology's nodes to the string's nodes. Using the Lightnet architecture the authors quantitatively prove that the user available throughput is increased up to nearly an order of magnitude without additional buffering requirements.

The authors in [14] formulate a method of designing the virtual topology while trying to minimize the network congestion and the average packet delay for a wavelengthrouted optical network. The objective is to minimize the maximum offered load in a logical link, given a set of contraints.

The authors in [6] also present a formulation for wavelength-routed optical networks. The main difference between the formulations in [14] and [6] is that the latter includes the wavelength constraints - the number of wavelengths available per link, and also the wavelength continuity constraint. The authors also give an alternative linear formulation which does not involve the non-linear terms that arise in the wavelength continuity constraint and de- lay minimization objective. The objective function based on delay is replaced with one that minimizes the average hop count. Under balanced flows the average hop count is inversely proportional to the network throughput. So the linear version of the formulation can be used to design a balanced network, such that the utilization of both the transceivers and wavelengths is maximized, thus reducing the cost of terminal equipment. The authors also analyze the trade-offs between switch sizes and terminal equipment and show that an improperly designed network could have a low utilization of one of these resources.

\subsection{Previous Work on Reconfiguration of WDM networks}

Reconfiguration in an optical WDM network is the process of rearranging the lightpath connections to dynamically optimize the network. The process of reconfiguration involves tearing down the existing lightpath connections that are no longer necessary, and setting up of new ones. A sample reconfiguration scenario is presented in Figure 3. The reconfiguration phase involves the transition from the first logical connection graph to the second. Traditionally reconfiguration of optical networks has been studied in two different contexts: broadcast optical networks [7], [10], [11] and wavelength-routed networks [6]. In [12] the authors propose a sequence of steps involved in the transition of an optical network from one configuration (virtual topology) to another. Reconfiguration involving no loss or delay of data is defined as hitless reconfiguration and studied in [15]. In this paper we restrict the problem of reconfiguration to wavelength-routed optical networks.

\subsubsection{Wavelength-routed Optical networks}

In [6], the authors provide a mathematical way of finding the best network configuration for the changed traffic demand in a wavelength-routed optical network. The formulation ensures that the new configuration is not too different from the existing configuration, thereby minimizing the number of re-tunings necessary. That is, if we can retain as many lightpaths (virtual links) as possible from the present topology and still satisfy the changed traffic requirements and generate a new optimized topology, we will be doing less reconfiguration (re-tunings).

In [12], the author proposes a minimally disruptive approach that transitions the network from one configuration to another through a sequence of branch exchange operations, so that only two links are disrupted at any given time. A branch-exchange operation can be defined as the swapping of destination nodes between two source-destination pairs.

\section{Problem description}

The physical topology is assumed to be generic, i.e., the nodes can be connected in arbitrary topologies using even multiple fibers between them. But the number of wavelengths carried on a fiber is constrained. Note that the analysis presented below is applicable only for a wavelengthrouted network which is used to carry packet traffic from a source to destination, perhaps, over several lightpaths. We 
do not consider optical packet switched networks, which provide an alternative implementation for carrying. packet traffic.

\subsection{Motivation}

Similar to the model given in [6], our model considers a network with an initial traffic matrix; reconfiguration analysis is done based on changes to this traffic matrix. The traffic matrix entries corresponding to row $i$ and column $j$ is the expected traffic from $i$ to $j$. We first determine the virtual topology that optimizes various metrics. We consider different objective functions like minimizing the number of lightpaths established, minimizing the number of hops (lightpath hops, same as in [6]), minimizing the number of physical links used to set up lightpaths, and a combination of all these objectives.

\subsection{Parameters}

Below we define the parameters used in the problem formulation.

Number of nodes in the network is $N$ (including both end nodes and switching elements).

Maximum number of wavelengths per fiber is $\mathrm{W}$ (a system wide parameter). Note that in the case of a bidirectional fiber, there would be $W$ wavelengths available in each direction.

Physical topology matrix $P$, where $P_{m n}$ denotes the number of fiber links interconnecting nodes $m$ and $n$. Note that for a network consisting of only bidirectional fibers the physical topology matrix would be symmetric i.e., $P_{m n}$ $=P_{n m}$. Also $P_{m m}=0$.

Fiber delay matrix $d$, i.e., fiber delays $d_{m n}$ from node $m$ to $n$, expressed as propagation delay, in time units (sec). For nodes that are not connected by a fiber link, the corresponding propagation delay is infinite.

Shortest path delay matrix $D$ where $D_{s d}$ denotes the sum of the delays on the shortest path between station $s$ and $d$ (in sec).

Lightpath length bound $\alpha$, bounds the delay over the path between nodes $i$ and $j$ in the virtual topology, w.r.t. the shortest path delay $D_{i j}$ between them. The maximum allowed delay being $\alpha \times D_{i j}$.

Number of transmitters and receivers at node $i$ are $T_{i}$ and $R_{i}$ respectively.

Traffic matrix $\Lambda_{s d}$ denotes the expected maximum rate of traffic flow from nodes $s$ to node $d$, with $\Lambda_{s s}=0$ for $s, d=$ $1,2,3 \ldots, N$ in units of bits/sec.

$C$ denotes the maximum capacity of a lightpath (in bits/sec).

Maximum loading (utilization) per channel $=\rho_{\max }, 0<$ $\rho_{\max }<1$. This restricts the queuing delay on the lightpath from getting unbounded by avoiding excessive link congestion.

\subsection{Variables}

In this section, we define the variables in the formulation. $v_{i j}$ denotes the number of lightpaths from node $i$ to $j$ in the virtual topology.

$p_{m n}^{i j}$ denotes the number of lightpaths between nodes $i$ and $j$ being routed through the fiber links between $m$ and $n$. $\lambda_{i j}^{s d}$ denotes the ratio of traffic flowing from node $s$ to node $d$ and employing a lightpath from $i$ to $j\left(0 \leq \lambda_{i j}^{s d} \leq 1\right)$.

\section{Linear Formulation}

Below, we present a linear formulation to solve the lightpath establishment and routing problem. The formulation is influenced by [6]. For a comparison of this method with some heuristic approaches, please refer to [6].

\subsection{Objective function(s)}

Minimize:

Obj1 (or $\lambda$ ):

$$
\frac{\sum_{s=1}^{N} \sum_{d=1}^{N} \sum_{i=1}^{N} \sum_{j=1}^{N} \lambda_{i j}^{s d} \times \Lambda_{s d}}{\sum_{s=1}^{N} \sum_{d=1}^{N} \Lambda_{s d}}
$$

Obj1 minimizes the average number of hops in the network encountered by a packet. This is the objective function used in [6].

Obj2 (or vp):

$$
\sum_{i=1}^{N} \sum_{j=1}^{N} v_{i j}+\sum_{i=1}^{N} \sum_{j=1}^{N} \sum_{m=1}^{N} \sum_{n=1}^{N} p_{m n}^{i j}
$$

Obj2 minimizes the total number of lightpaths set up in the network, as well as the number of physical links used. Obj3 (or $\lambda \mathrm{p}$ ):

$\frac{\sum_{s=1}^{N} \sum_{d=1}^{N} \sum_{i=1}^{N} \sum_{j=1}^{N} \lambda_{i j}^{s d} \times \Lambda_{s d}}{\sum_{s=1}^{N} \sum_{d=1}^{N} \Lambda_{s d}}+\sum_{i=1}^{N} \sum_{j=1}^{N} \sum_{m=1}^{N} \sum_{n=1}^{N} p_{m n}^{i j}$

Obj3 minimizes the hops as well as the number of physical links used.

Obj4 (or v $\lambda$ p):

$$
\begin{gathered}
\sum_{i=1}^{N} \sum_{j=1}^{N} v_{i j}+\frac{\sum_{s=1}^{N} \sum_{d=1}^{N} \sum_{i=1}^{N} \sum_{j=1}^{N} \lambda_{i j}^{s d} \times \Lambda_{s d}}{\sum_{s=1}^{N} \sum_{d=1}^{N} \Lambda_{s d}}+ \\
\sum_{i=1}^{N} \sum_{j=1}^{N} \sum_{m=1}^{N} \sum_{n=1}^{N} p_{m n}^{i j}
\end{gathered}
$$

Obj4 optimizes based on all the above three objective functions.

\subsection{Constraints}

Resource Constraints:

$$
\sum_{j=1}^{N} v_{i j} \leq T_{i} \forall i, 1 \leq i \leq N
$$

i.e., the number of outgoing (originating) lightpaths $\leq$ Number of transmitters at a node.

$$
\sum_{i=1}^{N} v_{i j} \leq R_{i} \forall j, 1 \leq i \leq N
$$


i.e., the number of incoming (terminating) lightpaths $\leq$ Number of receivers at a node.

$$
\sum_{m=1}^{N} p_{m k}^{i j}=\sum_{n=1}^{N} p_{k n}^{i j} \text { if } k \neq i, j
$$

i.e., the flow of traffic is conserved at intermediate nodes.

$$
\sum_{n=1}^{N} p_{i n}^{i j}=v_{i j}=\sum_{m=1}^{N} p_{m j}^{i j}
$$

i.e., the number of outgoing lightpaths from $i$ destined to $j$ $=$ the number of incoming lightpaths to $j$ from $i$.

$$
\sum_{i=1}^{N} \sum_{j=1}^{N} p_{m n}^{i j} \leq W \times P_{m n}
$$

i.e., the number of lightpaths in a link is bounded by the maximum available wavelengths in the link.

Integral Constraints:

$$
\begin{aligned}
\text { int } v_{i j} & \geq 0 \\
\operatorname{int} p_{m n}^{i j} & \geq 0
\end{aligned}
$$

Bifurcated Traffic flow: More than one virtual path can carry traffic from $s$ to $d$.

$$
\sum_{j=1}^{N} \lambda_{s j}^{s d}=\sum_{i=1}^{N} \lambda_{i d}^{s d}=1
$$

i.e., Conservation of traffic at the endpoints.

$$
\sum_{i=1}^{N} \lambda_{i k}^{s d}=\sum_{j=1}^{N} \lambda_{k j}^{s d} \text { if } k \neq s, d
$$

i.e., Conservation of traffic at the intermediate nodes/switches.

$$
\begin{aligned}
& \lambda_{i j}^{s d} \times \Lambda_{s d} \leq \rho_{\max } \times C \forall i, j, s, d 1 \leq i, j, s, d \leq N \\
& \sum_{s=1}^{N} \sum_{d=1}^{N} \lambda_{i j}^{s d} \times \Lambda_{s d} \leq \rho_{\max } \times v_{i j} \times C \forall i, j, 1 \leq i, j \leq N
\end{aligned}
$$

i.e., Constraint on $\lambda$ 's so that the total traffic carried by a link does not exceed its capacity.

$$
0 \leq \lambda_{i j}^{s d} \leq 1
$$

i.e., Constraint on $\lambda s$ that they should be less than the maximum possible.

Non-bifurcated traffic flow (if required): Only one sequence of lightpaths can carry traffic from $s$ to $d$. Instead of realvalued $\lambda_{i j}^{s d}$ s we would have:

$$
\text { int } \lambda_{i j}^{s d} \in\{0,1\}
$$

This would mean all traffic from $s$ to $d$ goes through a single sequence of lightpaths. It does not mean that a lightpath should carry only traffic for a single source-destination pair. Note that we do not handle non-bifurcated traffic flow in our experiments. However it can be incorporated easily by including the additional integral constraint on $\lambda_{i j}^{s d}$ (Eqn. 13).

Lightpath length bound

$$
\sum_{m=1}^{N} \sum_{n=1}^{N} p_{m n}^{i j} * d_{m n} \leq \alpha \times D_{i j} \times v_{i j} \forall i, j, 1 \leq i, j \leq N
$$

This ensures that a lightpath between two nodes cannot be longer than $\alpha$ times the shortest distance between those two nodes. Note that this equation does not consider the case in which there is more than one lightpath between two nodes, in which case one of them can be longer that $\alpha$ times the shortest distance between those two nodes. Later during the experimentation, we fix $v_{i j}$ to take binary ( 0 or 1 ) values, and that solves the problem. One other important observation is that it is possible for $p_{m n}^{i j}$ cycles to be formed in some cases, without violating any of the constraints (even for the formulation in [6]). The problem can be solved in the following ways:

- Restrict the values of $p_{m n}^{i j}$ to those corresponding to a few alternate paths between $i$ and $j$ (proposed in [6]).

- Always include $\sum p_{m n}^{i j}$ to be minimized in the objective function (used in our study).

In our study, we do not consider the wavelength continuity constraints to simplify the computation (by retaining linearity, as in [6]). However, in the post-processing phase, we ensure that solution satisfies the wavelength continuity constraint.

\section{Proposed Reconfiguration Algorithm}

In contrast to [6] our algorithm includes trade-offs between the amount of reconfiguration necessary and average packet delay. Packet delay is defined as the total propagation delay encountered by a packet from source to destination (Queuing delay is ignored). Average packet hop-count is defined as the average number of lightpath hops encountered by a packet from source to destination. This value is important in our analysis because of the fact that data might encounter delays due to electronic processing while hopping from one lightpath to another. We propose a new method to find out an optimal virtual topology for changed traffic demand. It is worth noting that the reconfiguration algorithm proposed here is independent of the actual method used to find the virtual topology. With slight modification, it can be applied to any solution. The following terms will be used in the discussion on reconfiguration:

Number of reconfiguration steps $(\Delta v)$ is given by the number of lightpaths that would have to be removed, as well as the new lightpaths that have to be established to realize the new solution. Mathematically, the number of reconfiguration steps $=\sum_{i j} \mid v_{i j}-v_{i j}[$ old $] \mid$.

Disruption $(\Delta p)$ quantifies the change at the physical layer (lightpaths and their routes) occurring in the network. In the formulation, it is the number of $p_{m n}^{i j}$ values affected. Mathematically, $\Delta p=\sum_{i, j, m, n} \mid p_{m n}^{i j}-p_{m n}^{i j}[$ old $] \mid$. 


\subsection{The method proposed in [6]}

The sequence of steps involved in the algorithm is as follows.

- STEP 1: Generate instances of the linear formulation, called $F($ old $)$ and $F(n e w)$, corresponding to traffic matrices $\Lambda_{s d}^{o l d}$ and $\Lambda_{s d}^{\text {new }}$ respectively.

- STEP 2: Solve the linear formulation for the old and new traffic respectively and record the solution $S($ old $)=$ $\left\{p_{m n}^{i j}[\right.$ old $], v_{i j}[$ old $]$, and $\lambda_{i j}^{s d}[$ old $\left.]\right\}, S($ new $)=\left\{p_{m n}^{i j}[n e w]\right.$, $\left.v_{i j}[n e w], \lambda_{i j}^{s d}[n e w]\right\}$, as well as the objective value for the new traffic demand $\Gamma$, and let $\Psi($.$) be the objective func-$ tion used (one of the four mentioned in our formulation). The algorithm given in [6] uses Obj1.

- STEP 3: Modify the linear formulation $F(n e w)$ to $F^{\prime}($ new) to include a new constraint

$$
\Psi(.)=\Gamma
$$

- STEP 4: The new objective function for $F^{\prime}(n e w)$ is

$$
\text { Minimize : } \sum \mid p_{m n}^{i j}-p_{m n}^{i j}[\text { old }] \mid
$$

Note that the above objective function is a non-linear function. To linearize it, we assume that $p_{m n}^{i j}$ 's can take only binary values. So the term $\mid p_{m n}^{i j}-p_{m n}^{i j}[$ old $] \mid$ is equal to $p_{m n}^{i j}$ if $p_{m n}^{i j}[$ old $]=0$. And $\mid p_{m n}^{i j}-p_{m n}^{i j}[$ old $] \mid$ is equal to $\left(1-p_{m n}^{i j}\right)$ if $p_{m n}^{i j}[o l d]=1$.

- STEP 5: Solve the new linear formulation $F(n e w)$. The new solution - the set of $p_{m n}^{i j}, v_{i j}$, and $\lambda_{i j}^{s d}$ involves setting up the least amount of new connections as well as de-commissioning of the unwanted previous connections, without increasing the objective function value.

\subsection{New Method : Reconfiguration as a Con-} straint

A useful metric for characterizing reconfiguration is the number of reconfiguration steps. Limiting the value of this metric (by specifying an upper bound) can enable network operators to limit the 'disruption' or 'down time' of the network during the transition from one virtual topology to another. The following method includes the number of reconfiguration steps as well as the disruption as constraints.

- STEP 1: Generate linear formulations $F($ old $), F(n e w)$ corresponding to traffic matrices $\Lambda_{s d}^{\text {old }}$ and $\Lambda_{s d}^{\text {new }}$ respectively (same as in [6]).

- STEP 2: Solve the linear formulation $F($ old $)$ for the old traffic and record the solution $S($ old $)=p_{m n}^{i j}[$ old $], v_{i j}[$ old $]$, and $\lambda_{i j}^{s d}[$ old $]$.

- STEP 3: Modify the linear formulation $F(n e w)$ to $F^{\prime}(n e w)$ by adding the new constraints

$$
\begin{gathered}
\sum_{i, j, m, n} \mid p_{m n}^{i j}-p_{m n}^{i j}[\text { old }] \mid \leq d_{\max } \\
\sum_{i, j} \mid v_{i j}-v_{i j}[\text { old }] \mid \leq r_{\max }
\end{gathered}
$$

where $d_{\max }$ is the maximum allowed value for disruption and $r_{\max }$ is the maximum allowable value for the number of reconfiguration steps.
- STEP 4: Solve the new linear formulation $F^{\prime}(n e w)$. The new solution - the set of $p_{m n}^{i j}, v_{i j}$, and $\lambda_{i j}^{s d}$ is the result of considering the new set of constraints.

\section{Results}

The experiments were conducted for a network with physical topology as shown in Figure 1. The CPLEX linear programming solver [9] was used to solve the linear formulations on a Sun Ultra-60 workstation. The linear formulation (without reconfiguration) with the following objective function $(\mathrm{Obj} 3)$ was used to solve the routing and lightpath establishment problem for the old (initial) traffic demand.

$$
\begin{gathered}
\text { Minimize : } \Psi=\frac{\sum_{s=1}^{N} \sum_{d=1}^{N} \sum_{i=1}^{N} \sum_{j=1}^{N} \lambda_{i j}^{s d} \times \Lambda_{s d}}{\sum_{s=1}^{N} \sum_{d=1}^{N} \Lambda_{s d}}+ \\
\sum_{i=1}^{N} \sum_{j=1}^{N} \sum_{m=1}^{N} \sum_{n=1}^{N} p_{m n}^{i j}
\end{gathered}
$$

In [6], the objective function was the same as Obj1. To reduce computation time we choose Obj3 over Obj4 (for our method), which has a high computation time due to the fact that different routes are tried to minimize the number of lightpaths set up. The lightpaths established according to the solution to the original formulation for the initial traffic matrix are recorded. The original formulation is now run on the new traffic matrix and the solution obtained. Data row 1 of Table 1 gives the solution obtained from solving the formulation $F(n e w)$ with Obj3. Data row 2 of Table 1 shows the results obtained from running the reconfiguration method proposed in [6] using Obj1 (Incidentally, we get the same results if we used $\mathrm{Obj3}$ for the method in [6]). From Data row 1 of Table 1 it can be seen that 10 reconfiguration steps would be necessary to transition from the solution for the old traffic matrix to the solution for the new traffic matrix (4 lightpaths removed and 6 lightpaths added). The $\Delta p$ column gives the disruption parameter for this reconfiguration. The average hop count and the average packet delay (measured in number of physical links traversed), corresponding to this solution, are also reported in row 1.

Data row 2 of Table 1 shows the results of running the method in [6] on the new traffic matrix. The stored solution for the old traffic matrix $\left(p_{m n}^{i j}[\right.$ old $], v_{i j}[$ old $], \lambda_{i j}^{s d}[$ old $]$ ) is used as the old values in this method. Due to the presence of an additional constraint in STEP 3 of the method in [6], the number of reconfiguration steps needed does not significantly decrease (Actually, in this case, the number of steps remain the same). Also note that the average packet delay does not change. This is because of the additional constraint in STEP 3.

The method proposed in this paper (Section 5.2) gives another approach to reconfiguration where we include the number of reconfiguration steps as well as the disruption as constraints and optimize based on the old objective function $(\mathrm{Obj} 3)$. The results obtained from such an approach, 
for the old and new traffic combinations, is given in Table 2. This approach provides more flexibility than the method in [6] in allowing us to specify an upper bound on the number of reconfiguration steps as well as the disruption value. Table 2 shows the results obtained from running the reconfiguration method proposed in this paper on the old and new traffic matrices, for different values of $r_{\max }$ and $d_{\max }$. The results show that it is possible to trade-off the average hop distance and packet delay for the number of reconfiguration steps. For example, with a $\Delta p$ value of 8 (as against 18 for the method in [6]) and a $\Delta v$ value of 5 (as against 10 for the method in [6]), the average hop distance is 1.176 (as against 1 for the method in [6]) and average packet delay is 1.823 (as against 1.588 for the method in [6]). These results show that for a small increase in the hop count and the packet delay, we can attain significant improvement in the number of reconfiguration steps. The decision to choose one solution over the other would depend on the absolute values of delays and the actual time it takes for a reconfiguration step to be performed.

The same experiment was conducted for a bidirectional ring network of size 8 and the results reported in Tables 3 . The results confirm the observations made from the previous experiment. The reconfiguration algorithm proposed here can be applied to any method used for generating the logical topology. The ease of including the constraints for different methods may vary.

\section{Conclusion}

In this paper we discussed a mathematically rigorous formulation for solving the lightpath reconfiguration problem in wavelength division multiplexed (WDM) optical networks. The formulation takes into consideration the fact that the traffic pattern in the network can be changing, and reconfiguration would have to be performed when the traffic pattern changes. We also explored the limitations of such a formulation and proposed different objective functions that can be used for optimization in finding the best virtual topology for a given traffic pattern. We proposed a modification to the reconfiguration algorithm proposed in [6], to include trade-offs between the amount of reconfiguration and the objective we are trying to optimize. We did not deal with the steps involved in detecting the need for reconfiguration i.e., when to trigger reconfiguration? Future work would include managing the reconfiguration process the actual process of transforming from one virtual topology to another.

\section{References}

[1] T. E. Stern and K. Bala, "Multi wavelength Optical Networks: A layered approach," Addison-Wesley Longman Inc., Mass., U.S.A, 1999.

[2] B. Mukherjee et al., "Some principles for designing a wide-area optical network," IEEE Trans. Networking, vol. 4, pp. 684-696, Oct. 1996.

[3] R. Ramaswami and K. N. Sivarajan, "Design of Logical Topologies for Wavelength-Routed Optical Net- works," IEEE J. on Sel. Areas in Comm., vol. 14, pp. 840-851, Jun. 1996.

[4] B. Mukherjee, "Optical Communication Networks," McGraw-Hill, New York, 1997.

[5] R. Ramaswami and K. N. Sivarajan, "Optical Networks: A Practical Perspective, Morgan Kaufman Publishers, Inc., San Francisco, CA, 1998.

[6] D. Banerjee and B. Mukherjee, "Wavelength-routed optical-networks: linear formulation, resource budgeting trade-offs, and a reconfiguration study," Proc., IEEE INFOCOM '97, pp. 269-276, Apr. 1997.

[7] J. -F. P. Labourdette, "Traffic optimization and reconfiguration management of multiwavelength multihop broadcast lightwave networks," Computer Networks and ISDN Systems, pp. 981-998, May 1998.

[8] I. Chlamtac, A. Ganz and G. Karmi, "Lightpath communications: An approach to high-bandwidth Optical WANs," IEEE Trans. on Comm., pp. 1171, vol. 40, Jul. 1992.

[9] ILOG CPLEX 6.5, "Reference Manual," ILOG S.A, March 1999.

[10] E. Baldine and G. N. Rouskas, "Reconfiguration and Dynamic Load Balancing in Broadcast WDM Networks," Photonic Networks Comm. J., vol. 1, pp. 4964, Jun. 1999.

[11] E. Baldine and G. N. Rouskas, "Dynamic Reconfiguration Policies for WDM Networks," Proceedings of IEEE INFOCOM '99, pp. 313-320, Mar. 1999.

[12] J. -F. P. Labourdette, G. W. Hart and A. S. Acampora, "Branch-Exchange Sequences for Reconfiguration of Lightwave Networks," IEEE Trans. on Comm., vol. 42, Oct. 1994.

[13] I. Chlamtac, A. Ganz, and G. Karmi, "Lightnets: Topologies for High-Speed Optical Networks," IEEE/OSA J. of Lightwave Tech., vol. 11, May/Jun 1993.

[14] R. Ramaswami, and K. N. Sivarajan, "Design of Logical Topologies for Wavelength-Routed Optical Networks," IEEE J. on Sel. Areas In Comm., vol. 14, Jun. 1996.

[15] K. Bala, G. Ellinas, M. Post, and C. -C. Shen, "Towards Hitless Reconfiguration in WDM Optical Networks for ATM Transport," IEEE/IEICE Global Telecom. Conf., pp. 316, 1996.

[16] T. E. Stern, and K. Bala, "Multiwavelength Optical Networks: A Layered Approach," Addison Wesley Longman, Inc., Reading, Massachusetts 01867, 1999.

[17] J. P. Jue, and B. Mukherjee, "Multiconfiguration Multihop Protocols (MMPs): A New Class of Protocols for Packet-Switched WDM Optical Networks," Proc., IEEE INFOCOM '98, pp. 816, 1998. 


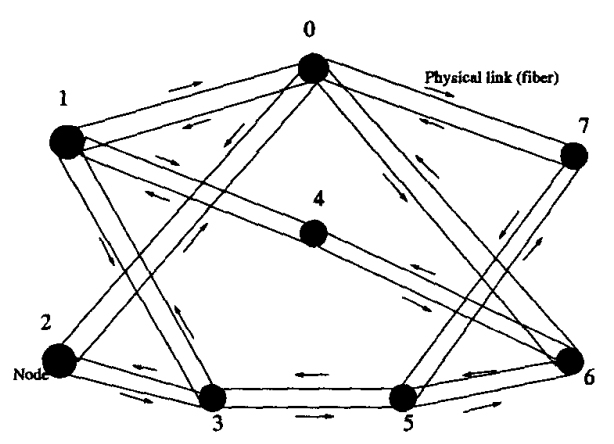

Figure 1: The network physical topology. A directed edge connecting two nodes represents a unidirectional fiber link.

\begin{tabular}{|c|c|c|c|c|c|}
\hline $\begin{array}{c}\text { Method } \\
\text { used }\end{array}$ & $\begin{array}{c}\text { comp. } \\
\text { time } \\
\text { (secs) }\end{array}$ & $\begin{array}{c}\text { Disru- } \\
\text { ption } \\
\Delta p\end{array}$ & $\begin{array}{c}\text { \# of rec- } \\
\text { figuration } \\
\text { steps }(\Delta v)\end{array}$ & $\begin{array}{c}\text { Avg } \\
\text { hop } \\
\text { count }\end{array}$ & $\begin{array}{c}\text { Avg } \\
\text { pkt } \\
\text { delay }\end{array}$ \\
\hline $\begin{array}{c}\text { Using Obj2 } \\
\text { w/o } \\
\text { reconf. }\end{array}$ & 2.14 & 18 & 10 & 1 & 1.59 \\
\hline $\begin{array}{c}\text { Method } \\
\text { in [6] } \\
\text { using Obj1 }\end{array}$ & $7.63^{*}$ & 18 & 10 & 1 & 1.59 \\
\hline
\end{tabular}

Table 1: Table showing number of reconfiguration steps, disruption the average packet delay, average hop count for the new traffic over network of Figure 1. The solution for the old traffic demand consisted of 17 lightpaths. Computation done on a lightly loaded Sun Ultra-60 workstation using CPLEX. $\left(^{*}\right)$ - actual time taken $=7.63+2.14=$ 9.77 secs.

\begin{tabular}{|c|c|c|c|c|c|c|}
\hline$d_{\max }$ & $r_{\max }$ & comp. & Actual & Actual & $\begin{array}{c}\text { Avg. } \\
\text { hop } \\
\text { count }\end{array}$ & $\begin{array}{c}\text { Avg. } \\
\text { pkt. } \\
\text { delay }\end{array}$ \\
\hline 5 & 4 & 12.90 & 5 & 4 & 1.33 & 2.65 \\
\hline 6 & 4 & 11.59 & 6 & 4 & 1.24 & 2 \\
\hline 10 & 6 & 12.01 & 8 & 5 & 1.18 & 1.83 \\
\hline 12 & 6 & 12.01 & 10 & 5 & 1.18 & 1.88 \\
\hline 18 & 10 & 7.78 & 16 & 9 & 1.06 & 1.59 \\
\hline 22 & 12 & 6.40 & 20 & 12 & 1 & 1.59 \\
\hline
\end{tabular}

Figure 2: Difference between physical link, lightpath and virtual path.

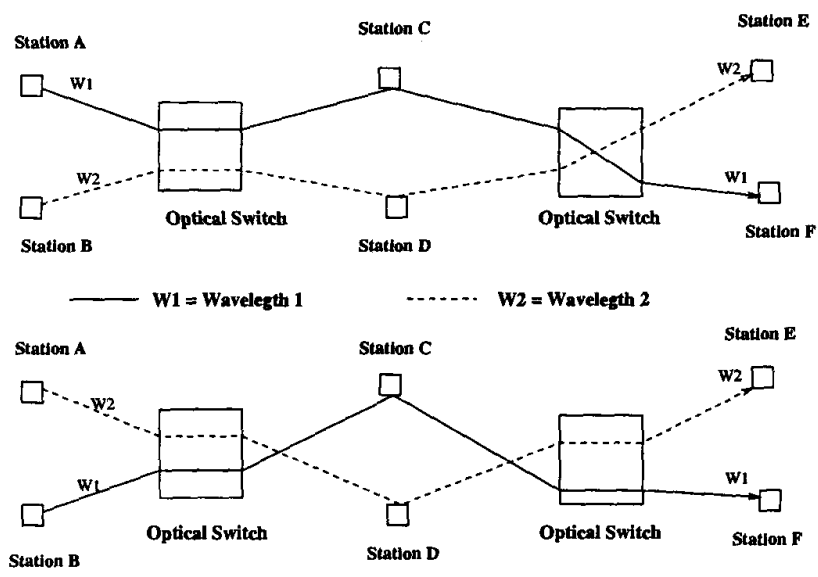

Figure 3: A reconfiguration scenario. Note that the transmitting and receiving wavelengths are different in the two cases.
Table 2: Table comparing the results obtained from the new reconfiguration algorithm using $\mathrm{Obj} 3$ for different values of $d_{\max }$ and $r_{\max }$ for the network in Figure 1 . The solution for the old traffic consisted of 17 lightpaths. Computation done on a lightly loaded Sun Ultra-60 workstation using CPLEX.

\begin{tabular}{|c|c|c|c|c|c|c|c|}
\hline $\begin{array}{c}\text { Meth. } \\
\text { used }\end{array}$ & $d_{\max }$ & $r_{\max }$ & comp. & Act. & Act. & $\begin{array}{c}\text { Avg. } \\
\text { hop } \\
\text { (secs) }\end{array}$ & $\begin{array}{c}\text { Avg. } \\
\text { pkt. } \\
\text { delay }\end{array}$ \\
\hline No reconf. & - & - & 693.11 & 26 & 16 & 1.24 & 2.69 \\
\hline$[6]$ & - & - & $885.37^{*}$ & 26 & 16 & 1.24 & 2.75 \\
\hline New & 14 & 8 & 19.89 & 13 & 8 & 1.37 & 2.69 \\
\hline New & 15 & 8 & 28.32 & 13 & 8 & 1.37 & 2.88 \\
\hline New & 17 & 9 & 48.13 & 15 & 9 & 1.30 & 2.94 \\
\hline New & 20 & 12 & 97.54 & 20 & 11 & 1.32 & 2.94 \\
\hline
\end{tabular}

Table 3: Table comparing the results obtained from the new reconfiguration algorithm for different values of $d_{\max }$ and $r_{\max }$ using $\mathrm{Obj} 3$, for the bidirectional ring network (8 nodes, a bidirectional fiber between two nodes with maximum of 2 wavelengths in each direction). The solution for the old traffic consisted of 18 lightpaths. Computation done on a lightly loaded Sun Ultra-60 workstation using CPLEX. $\left({ }^{*}\right)$ - actual time taken $=885.37+693.11=1578.48$ secs. 